\title{
Volume Estimation from Uncalibrated Views Applied to Wound Measurement
}

\author{
B. Albouy ${ }^{1}$, S.Treuillet ${ }^{2}$, Y. Lucas ${ }^{1}$, and J.C. Pichaud ${ }^{3}$ \\ ${ }^{1}$ Laboratoire Vision \& Robotique, Université d'Orléans \\ ENSI, 10 Bd Lahitolle 18000 Bourges, France \\ Benjamin. Albouy@ensi-bourges.fr, \\ Yves. Lucas@bourges . univ-orleans. fr \\ ${ }^{2}$ Polytech'Orléans, Site Galilée, 12 rue de Blois, BP 6744, 45067 Orléans, France \\ Sylvie.Treuillet@univ-orleans.fr \\ ${ }^{3}$ Hôpital La Tour Blanche, Issoudun, France \\ jc.pichaud@wanadoo.fr
}

\begin{abstract}
The aim of the ESCALE project is to supply the medical staff with objective and accurate 2D and 3D measurements for wound healing assessment from color images acquired in a free manner with a low cost digital camera. The problem addressed in this paper is the volume estimation from uncalibrated views. We present two experimentations. A Monte Carlo simulation on synthetic perturbated data leads to an average error of $3 \%$ on reconstructed points. Then, triangulation based volume estimation obtained from two uncalibrated real images gives us hope that an accuracy less than $5 \%$ is achievable. So this technique is suited to accurate wound 3D measurements. Combined with true color image processing for colorimetric tissue assessment, a such low cost system will be appropriate tool for diagnosis assistance and therapy monitoring in clinical environment.
\end{abstract}

\section{Introduction}

Pressure ulcers or bed sores, are damages of the skin affecting people confined to bed or wheelchair, exposed to prolonged pressure on bony prominence, such as the buttocks and heels. These wounds may appear in a few hours but the healing process can last from two weeks (30\% of cases) to several years. Whether pressure sores occur or not depends on patient related factors. The most important of these factors are age, neurological condition, dietary status, blood circulation and skin moisture degree. Several surveys reveal an average of prevalence between $15 \%$ and $20 \%$ among hospitalized patients. Within the E.U., at least 2 million persons suffer from chronic skin wounds, such as pressure ulcers or diabetic lesions. Health care costs attributed to chronic skin wounds are estimated at 8 billions euros per year and the number of ulcers is expected to increase by over $25 \%$ by the year 2010 , as a consequence of the growing part of aged population.

The effective management of non healing wounds is based on a complete patient history, a detailed initial assessment of the wound, and an analysis of probable causative factors. This information is used to personalize a management strategy to the underlying pathophysiology preventing healing and to implement appropriate 
wound interventions. Regular reassessment of healing progress and medical care changes are also necessary. In [1], the authors review clinically useful wound measurement approaches, and encapsulate key wound parameters in the simple mnemonic, which begins by MEASURE: length, width, depth, and area. But quantitative and accurate wound measurements remain a substantial challenge, because clinical practices are currently limited to qualitative visual evaluation [2]. Sometimes wound area measurement is carried out from acetate manual tracing, more rarely alginate moulds are used for measuring volume. All these methods are painful for the patient and subject to contamination, irritation or allergic reaction by a contact with the wound site. Following upon this lack of effective metric to evaluate the wound healing, the investigation and progress in wound treatment are limited. Hence, there is a demand from the medical staff for a convenient, accurate, reproducible and non-contact method to achieve wound periodic assessment and therapeutic monitoring.

\subsection{Relative Works}

To address this problem, a variety of techniques based on image processing have been explored since ten years [3-10]. The first works started from single pictures and carried out multi-center clinical studies to compare wound area measurements obtained by computerized imaging techniques (digitalized pictures) and acetate tracings. Another way investigates color analysis for skin healing assessment and kinetics [3,4]. But 2D measurements are not sufficient for a complete wound assessment [1]. Because wound healing frequently occurs through changes in depth rather than surface area, 3D measurements are necessary.

Plassmann and Jones [5, 6] pioneered a structured light technique with a system called MAVIS (Measurement of Area and Volume Instrument System): a set of colorcoded stripes is projected onto the wound area and recorded by a CCD camera at an angle about 45 degrees. They used a plaster model to achieve measurements with a mean error of less than $5 \%$ (if the wound area is greater than $9 \mathrm{~cm}^{2}$ and less deep than $3 \mathrm{~cm}$ ). Nevertheless, the precision of results from wound models, undertaken as part of a validation trial of the tool, could not achieved during clinical practice. However, MAVIS was consistently more precise than other methods used, it was noted to have high precision in wounds with larger dimensions, but it was of no value in large, circumferential, deep or under-mining wounds. Krouskop [9] used also structured light grid pattern of 16x16 dots captured by a digital camera to calculate the area and volume of wound. Image processing takes less than 5 minutes for a trained computer operator. Volume precision has been estimated on plaster molds with spherical indentation. Results indicate a precision within $\pm 3 \%$, when at least 144 of pattern dots lie within the wound.

A second approach was developed with MEDPHOS (Medical Digital PHOtogrammetric System) in [7, 8]: three cameras are mounted on a triangular frame in a slightly convergent way. The set-up includes also additional light source and texture projection in the center. The distance between cameras is fixed to $15 \mathrm{~cm}$ and implies a distance to the wounds of about $30 \mathrm{~cm}$. The calibration step is critical for the accuracy of the digital model and involves a trained operator. Experiments with patients were carried out on different types of wounds. This method sometimes failed due to many specular reflections caused by moisture on the wound surface, and the 
main goal of next works has been to improve the robustness. Finally, DERMA system [10] uses a Minolta VI910 scanner to acquire the 3D geometry of the wound and to capture a RGB image aligned to the geometry with 640x480 pixels resolution. In conclusion, we can observe that all previous systems are expensive and cumbersome equipment, not really suitable in clinical environment. Furthermore these dedicated vision systems fail in measuring very large wounds and their lack of portability is a severe drawback for imaging not easily accessible wounds without uncomfortable position for the patients.

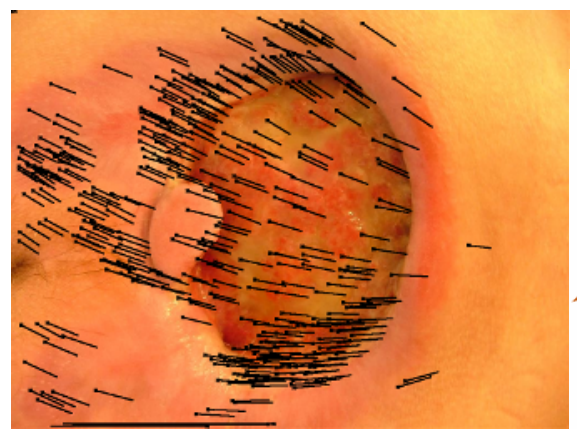

(a)

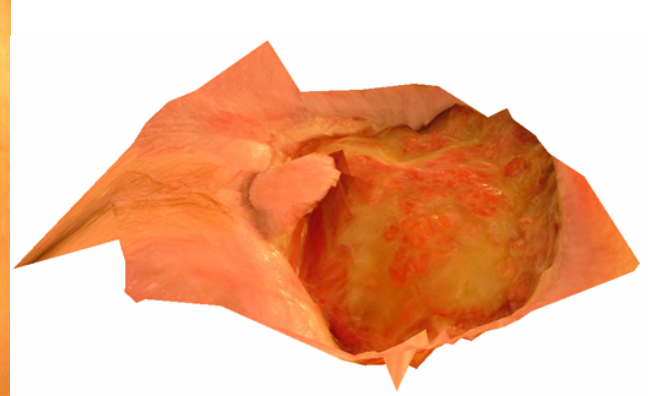

(b)

Fig. 1. The 3D color model of a real wound may be obtained from two uncalibrated views. (a) Left view with about four hundred inliers selected by MAPSAC method, (b) Color texture mapped on 3D model obtained by Delaunay triangulation.

\subsection{Escale Project}

The aim of ESCALE project is to develop image processing integrated tools to provide accurate 2D and 3D measurements of wounds from a series of color pictures (Fig.1). Unlike previous works, we have favored a portable, fast and safe technique for the patient. So, we prefer to use a standard digital camera to provide the medical staffs with a handy low cost image acquisition system. Digital camera is now a widespread device which delivers very high resolution color images 3 Mpixels or more), with zoom facility to adapt the field of view on a large variety of wound dimensions (from 4 to $400 \mathrm{~cm}^{2}$ ). The portability is also useful for tele-care purpose with home nursed patients by internet image transfer.

Behind this easy and free image capture, real technical difficulties are dissimulated, such as unknown geometry views and varying lighting conditions. So, we develop advanced techniques concerning $3 \mathrm{D}$ uncalibrated reconstruction and color segmentation. The problem addressed in this paper is the volume estimation from uncalibrated views. The subject of $3 \mathrm{D}$ reconstruction from a series of images is a widely studied topic in computer vision community. But what about the final triangulation accuracy that could be obtained on 3D points from two uncalibrated views? And what volume precision could we hope for? This subject is not really documented because uncalibrated reconstruction is generally devoted to $3 \mathrm{D}$ visualization or augmented reality from video sequences [11]. The reconstruction chain implemented is described in the next section. A Monte Carlo simulation provides error estimation to compare the influence of the fundamental matrix 
estimation algorithms in $3 \mathrm{D}$ point reconstruction. Next, the volume estimation obtained from triangulation on real images is presented with experimental results in section 3. Finally, we conclude and propose further improvements.

\section{Reconstruction Error from Uncalibrated Views}

Two images taken from different points of view allow the reconstruction of 3D positions thanks to a triangulation algorithm. But without information on geometry between the two views, a self-calibration step is needed before to run a triangulation algorithm. The 3D reconstruction chain starts with point correspondences on the two images. Several thousands of interest points (corners) are detected in each color image by the Harris \& Stephen algorithm adapted by Gouet [12]. As usual, this detection is corrupted by noise. Next, point matching is realized by cross correlation using a local similarity function based on textured neighbourhood of each corner. Unfortunatly, this process provides a lot of correspondence mismatches. These outliers may be extracted by applying MAPSAC method [13]. This method computes the fundamental matrix from a random set of seven corner correspondences and tests all other matches against the epipolar constraint. A number of consistent correspondences is obtained by applying a threshold distance to epipolar lines. This step is repeated for many random sets and the matrix obtaining the largest number is accepted. The outcome is a set of inliers (Fig.1a). The fundamental matrix is then replaced by its closest rank-2 matrix by using singular value decomposition before to apply self-calibration based on Kruppa equations [14].

To simplify the computation of the intrinsic parameters, we apply some common assumptions (squared pixels and same focal length for all the views). Then, extrinsic parameters are calculated and it is possible to reconstruct the $3 \mathrm{D}$ scene using a classical triangulation method. But, as the geometry is only defined up to a scale factor, one distance must be known on the real world to achieve Euclidian measurements on the reconstructed data. Performance characterization of this reconstruction chain can be accessed in [15]. To estimate the error on 3D points reconstruction, a Monte Carlo simulation involves an iterative process using randomly noised data inputs. Several hundred of 3D points are randomly generated and projected in the two image plans under a known geometry. To model the effect of noisy image detection, Gaussian noise has been added on the image projections. Mismatches between images have also been simulated.

Five fundamental matrix estimation algorithms have been compared in the global reconstruction chain presented above (Fig.2). It appears that 8-points method is the most robust for noisy image inputs, and that MAPSAC is the most robust for mismatches. So, the two methods have been mixed: MAPSAC is used to classify good and bad correspondences, whereas 8-points algorithm is used to compute the fundamental matrix from resulting inliers. On these synthetic data, 3D reconstruction mean error is less than $3 \%$ with regards to a good triangulation configuration (60 degree angle between the two views). Applying this reconstruction chain on real images allow to obtain triangulation and color texture mapped 3D model (Fig.1b). Pictures are compressed and the performance of this algorithm under JPEG image degradation has already been tested by Torr [16]. It is suggested that $Q=70$ is the most an image should be compressed without risk of substantial degradation. So using 
compressed images is not a problem. Volume estimation obtained from triangulation on real images is presented in next section.

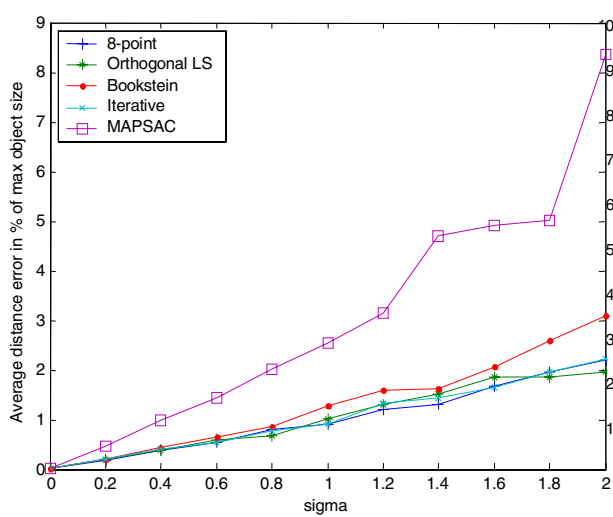

(a)

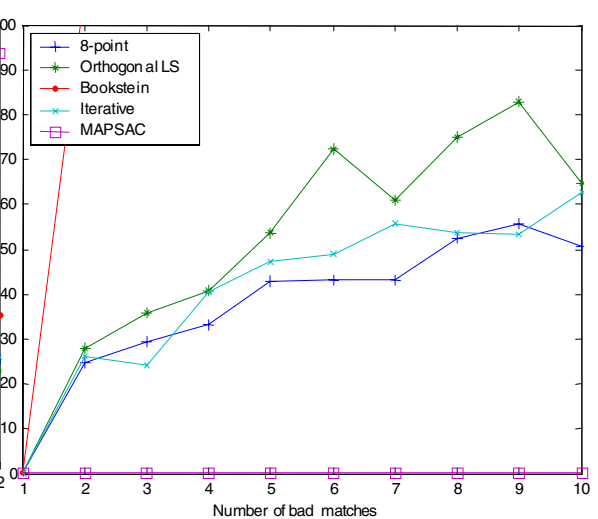

(b)

Fig. 2. Performance evaluation of fundamental matrix estimation algorithms by a Monte Carlo simulation. The y-axis gives the average distance error between reconstructed points and reference data, relative to (a) standard deviation of the Gaussian noise added on positions of projected points (b) number of mismatches with a standard deviation of Gaussian noise fixed to unity $(\sigma=1)$.

\section{Triangulation Based Volume Estimation}

Delaunay triangulation is the leading method for generating surface from 3D points. This triangulated surface may also be used for volume estimation [17]. The volume calculation consists in summing the elementary volumes under each prism formed by facets and their orthogonal projections on a reference plane. This plane, $P$, corresponding to the complete healing of healthy skin, is the mean plane in least square sense computed from points on the wound bounds. The equation for $P$ is calculated in the camera reference frame. Once this plane is known, a rigid transformation $H$ is applied to reconstructed data in order to fit the reference plane with the canonic plane $P_{r e f}: z=0$. So, the definition of $H$ is $P_{r e f}=k H \cdot P$, where $k$ is a scale factor due to homogeneous coordinates. The rigid transformation $H$ includes two rotations around $x$-axis and $y$-axis, and a translation $t_{z}$ along $z$-axis. These four parameters $\left(\theta_{x} \theta_{y} t_{z} k\right)$ are determined by analytical equations.

In order to achieve measurements with a mean error, repetitive tests have been done on a cork wound model over several pairs of images from different points of view (Fig.3). A pair of colored balls with known distance is placed in the field of view to provide a metric reference. These balls are automatically detected in the images. The contour of the wound is roughly drawn by manual selection. The ground truth is given by the mean of twenty successive weighting the water inner filling on a balance with a centigram precision. Volume estimations have been realized on sixteen pairs of images. Results are reported in Table 1. Comparison of theses estimations against the ground truth is given in Table 2 . The triangulation based volume is slightly overestimated with a percentage error around $3 \%$. 
Even if this reconstruction accuracy is sufficient for wound volumetric estimation, these preliminary results raise two difficulties. The first one is the uncertainty of reconstruction. It highly depends on the angle between the triangulation rays that is the movement between the images. Points are less precisely localized along the ray as the rays become more parallel (forward motion in particular). The reconstruction error is large for high noise level, since there is a little movement between images. Uncertainty of measurements may be noticed in Table 1: the standard deviation to mean ratio rises up to $13 \%$. The best configuration is obtained for a 90 degrees angle between views. But in this case, it will be very difficult to achieve reliable matching. This explains why published research [11] is based mostly on video sequences to gradually go over distant points of views through successive close images. A second difficulty derives from singularities that could lead to an insolvable self calibration [18]. This is the case for orbital movement (pure rotation by going round the object), for example. Further attention needs to be given to the image capture protocol, in particular by taking more than two views.

Table 1. Volume estimation on sixteen pairs of images

\begin{tabular}{|l|l|l|l|l|l|l|l|l|}
\hline \multirow{2}{*}{ Measurements } & 44.40 & 41.70 & 34.60 & 44.27 & 40.71 & 40.58 & 43.90 & 33.80 \\
\cline { 2 - 8 } & 41.60 & 40.61 & 30.86 & 37.43 & 54.15 & 40.63 & 39.68 & 40.37 \\
\hline
\end{tabular}

Table 2. Comparison of the triangulation based volume estimation against ground truth

\begin{tabular}{|r|c|c|c|c|c|}
\cline { 2 - 5 } \multicolumn{1}{c|}{} & Mean & $\begin{array}{c}\text { Standard } \\
\text { deviation }\end{array}$ & Min & Max & $\begin{array}{c}\text { St. dev./M } \\
\text { ean }\end{array}$ \\
\hline Estimated volume & 40.58 & 5.25 & 30.85 & 54.15 & $12.93 \%$ \\
\hline Ground truth & 39.20 & 1.03 & 37.38 & 41.10 & $2.63 \%$ \\
\hline
\end{tabular}

\section{Conclusion and Future Works}

For a vision module to be of practical use for volume measurements, its performance must be evaluated. This paper present two experimentations. First a 3\% error rate is observed on reconstructed points by a Monte Carlo simulation on synthetic perturbated data. Next, a volume estimation based on uncalibrated triangulation on real images suggests that an accuracy less than 5\% may be achievable. So this technique is suited to clinical wound 3D measurements. Taking into account the natural curvature of the body in the 3D surface of a healing wound will increase the quality of volume estimation. Several improvements are currently implemented for the matching step, a major difficulties in image processing, like using geometry invariants and relaxation. In order to be of general practical use over a wide variety of conditions and to avoid self calibration singularities, more views will be used to refine triangulation. Combined with true color image processing for quantitative colorimetric tissue assessment, such a low cost system will be a convenient tool for diagnosis assistance and therapy monitoring in clinical environment. 


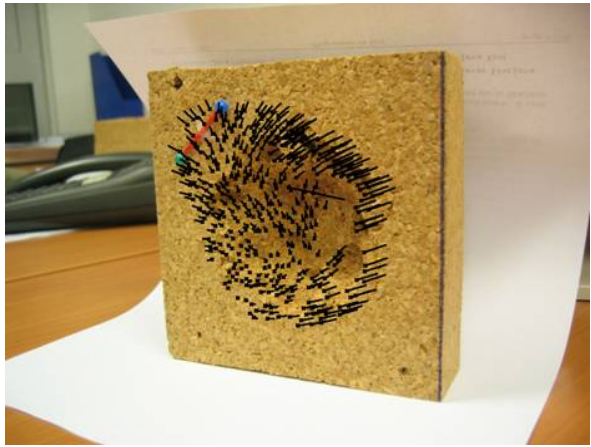

(a)

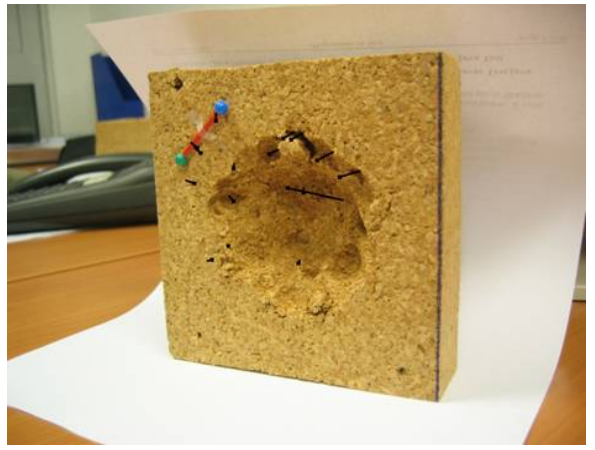

(c)

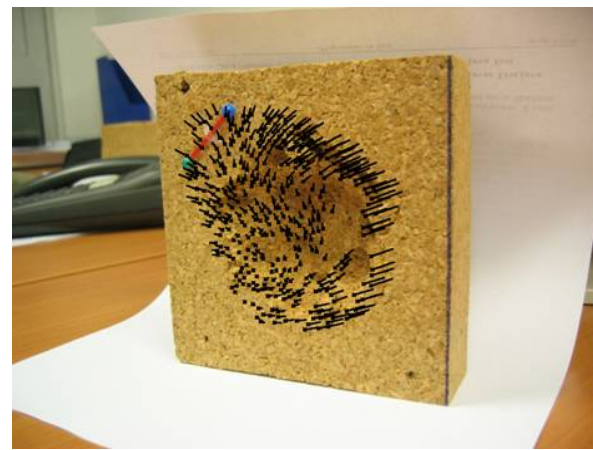

(b)

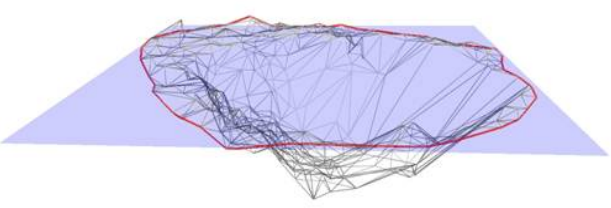

(d)

Fig. 3. A cork model of wound is used to estimate the accuracy of triangulation based volume calculation. (a) All matches obtained on image, (b) inliers, (c) outliers, (d) triangulated surface with wound bound and reference plan.

\section{Acknowledgement}

This research was supported by the french Ministry of Research and the European Social Found through their delegations in Centre area.

\section{References}

1. Keast D.H., \& al.: MEASURE: A proposed assessment framework for developing best practice recommendations for wound assessment. Wound Repair and Regeneration, June Vol.12 (2004) s1-s17

2. Pudner Rosie: Measuring wounds. (2002) http://www.jcn.co.uk/

3. Herbin M., Bon F.X., Venot A., Jenlouis F., Dubertret M.L.: Assessment of healing kinetics through true color image processing. IEEE Transactions on Medical Imaging 12-1 (1993) 39-43

4. Bon F.X., Briand E., Guichard S., Couturaud B., Reval M., Servant J.M. and Dubertret L.: Quantitative and kinetic evolution of wound healing through image analysis. IEEE Transactions on Medical Imaging 19-7 (2000) 767-772 
5. Plassmann P., Jones B.F., Ring E.F.J.: A structured light system for measuring wounds. Photogrammetric Record Vol.15 Nº6 (1995) 197-203

6. Plassman P., Jones T.D.: MAVIS: a non-invasive instrument to measure area and volume of wounds. Med. Eng. Phys 20-5 (1998) 332-338

7. Boersma S.M., Van den Heuvel F.A., Cohen A.F., Scholtens R.E.M.: Photogrammetric wound measurement with a three-camera vision system. IAPRS Vol. XXXIII Amsterdam (2000)

8. Malian A., Heuvel Van Den F.A., Azizi A.: A Robust Photogrammetric System for Wound Measurement. ISPRS Symposium commission V Corfu Greece International Archives of Photogrammetry and Remote Sensing Vol. 34 Part 5 (2002) 264-269

9. Krouskop T.A, \& al.: A non contact wound measurement system. Journal of Rehabilitattion R\&D Vol. 39 N³ May/June (2002) 337-346

10. Romanelli M., \& al.: Technical advances in wound bed measurement. WOUNDS (2002) $14-58$

11. Pollefeys M. : Self-Calibration and Metric 3D Reconstruction From Uncalibrated Image Sequences. PhD Thesis of the Catholic University of Leuven (1999)

12. Gouet V.: Mise en correspondance d'images en couleurs, application à la synthèse de vues intermediaires, Thèse de Doctorat de l'Université de Montpellier II Sciences et Techniques du Languedoc (2000)

13. Torr P.H.: Bayesian Model Estimation and Selection for Epipolar Geometry and Generic Manifold Fitting. International Journal of Computer Vision 50-1 (2002) 35-61

14. Sturm P.: On Focal Length Calibration from Two Views. Conference on Computer Vision and Pattern Recognition, vol. II (2001) 145-150

15. Albouy B., Treuillet S., Lucas Y. Birov, D.: Fundamental matrix estimation revisited through a global 3D reconstruction framework. IEEE ACIVS Bruxelles (2004) 185-192

16. Torr P.H, Zisserman A.: Performance characterization of fundamental matrix estimation under image degradation. Machine Vision \& Application Vol.9 (1997) 321-333

17. De Floriani K., Puppo E.: An on-line algorithm for Delaunay triangulation. Graphic Models and Image processing Vol.54 N³ (1992) 290-300

18. Sturm P. : Vision 3D non calibrée : Contributions à la reconstruction projective et études des mouvements critiques pour l'auto-calibrage. Thèse de Doctorat au Laboratoire GRAVIR - IMAG de l'INRIA Rhône-Alpes (1997) 